\title{
DEVELOPMENT OF A BLOOD ANALOG FOR THE HEMODYNAMIC EFFICIENCY EVALUATION OF CARDIOVASCULAR DEVICES
}

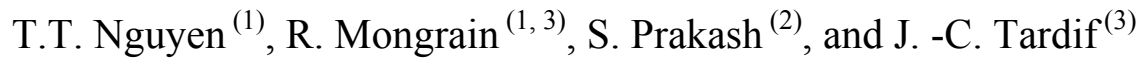 \\ (1) Department of Mechanical Engineering, McGill University \\ (2) Department of Biomedical Engineering, McGill University
}

(3) Montreal Heart Institute

\begin{abstract}
Accurate simulation of blood hemolytic potential (i.e. the destruction of red blood cells) would be a valuable tool to evaluate blood-interacting devices. Indeed, such a model would alleviate several practical problems with manipulating real blood (addition of anticoagulants and antibiotics, filtration, etc.). In the present study, we have developed a blood analog which is a suspension of micron-sized natural polymeric gel particles, each encapsulating a dye agent. These particles release the dye agent through shear-induced diffusion and fragmentation from exposure to high, non-physiological shear rates. A controlled shear field was applied to blood analog samples using a custom-made grooved parallel plate geometry device as previously reported in the literature. The device was designed to apply a shear stress and shear exposure duration in the range of 0.15 - 269.18 Pa and 156 - 1297 ms, respectively. From the results obtained, a correlation between the human index of hemolysis and the concentration of the released dye agent can be found. Therefore, the use of the proposed suspensions appears suitable as a blood analog for the in vitro evaluation of the destructive effects of cardiovascular devices.
\end{abstract}

Keywords: Mechanical hemolysis, blood analog, microparticle diffusion, shear stress, time exposure

\section{Introduction}

Since the early observation that patients with prosthetic cardiovascular devices (artificial heart valves, ventricular assist devices) developed significant hemolytic anemia, a great number of studies that span over half a century have been committed to understand how the erythrocytes respond structurally and morphologically to non-physiological levels of pressure, temperature, shear stress, and duration of exposure. A first empirical relation to fit shear stress and duration of exposure can be found in Rand [1]:

$$
\tau^{2} \cdot t_{\exp }=\text { constant }
$$

However, this relation was only used for qualitative experiments, as done by Papantonis [2] for radial flow impellers. A first attempt at a quantitative relation for the index of hemolysis was then derived by Lambert [3] with the following relation:

$H I \cong \tau \cdot t_{\mathrm{exp}}^{0.5}$

A similar development was later conducted by Wurzinger et al using a custom coaxial cylinders viscometer [4, 5] yielding the following relation presented by Giersiepen et al $[4,6]$.

$H I(\%)=3.65 \times 10^{-5} \cdot \tau^{2.416} \cdot t_{\exp }^{0.785}$

The difference between relations (2) and (3) is very significant and is assumed to be caused by subhemolytic damages, or predamage, to the red cells during the preparation of samples making it sensitive to shear stress values $(\tau)$ (higher exponent)[7].

The equation developed by Giersiepen et al is the most used relation for CFD studies involving hemolytic potentials. However, there is significant differences between the blood preparation protocols and thus, efforts are being done towards this end [7, 8]. One important limitation lies in the fact that human blood cannot be obtained in large quantities to provide enough data to derive a reliable reference. In that context, blood analogs would provide the means to develop a standard protocol. 
A commonly-used substitute is blood derived from other species, which have been employed in rheological and hemolysis studies as well as in the evaluation of many cardiovascular devices [8-10]. Although the chosen species from which blood is extracted do not share the same genus as humans', their physiological and mechanical properties bear many similarities such as viscosity, cell shape and size. Moreover, an important factor that would obviously influence the choice of a blood analog would be the difference in its mechanical fragility with respect to human blood. For example, a study led by Jikuya et al (1998) [11] showed that, in addition to differences in mean corpuscular volume between ovine, bovine and human, bovine blood showed significantly lower damage (about 50\%) compared to human blood while ovine blood displayed higher damage values (about 180\%).

Furthermore, separate reviews from Mueller et al [12] and Thurston [13] underlined the major problems that still influence results when using analogs derived from animals. They are tied to the fact that these blood analogs are biological. Therefore, there is great variability between samples, high chances of induced subhemolytic damages through manipulation and storage, and unknown distributions of cell age in samples. Other means have therefore been developed to solve the biological problem such as creating an artificial blood analog through the use of polymers that have controllable and reproducible characteristics. One such analog has been introduced by Pohl et al [9, 10] which exploits the changes in low shear viscosity plateau values of a solution of Aqueous Polyacrylamide after being exposed to high shear stresses for a determined length of time. In principle, a dispersed polymer follows a series of rearrangements of its molecular network caused by increasing shear stress. Thus, applying stresses beyond a certain range causes an irreversible degradation of the network and can be directly quantified by measuring the decrease in the low shear rate viscosity plateau of the blood analog. Another recent approach to develop an artificial blood analog for hemolysis has been developed by Maruyama et al $[8,14]$ which consisted of a suspension of microcapsules that ruptured under high shear stresses and liberated an agent that reacts to the suspending medium, leuco dye (colorless form of cyan dye). The reaction produced a low red contrast that is used for direct quantification. A thorough analysis was made by the same author assessing the influence of different capsule membrane materials (melamine and urethane) and capsule sizes. The suspension was found to be Newtonian and only a specific size of the microcapsules gave satisfactory results. Furthermore, since the authors used a tissue grinder to induce fragmentation, the blood analog was not tested to study the effect of time exposure on its hemolytic properties.

In this work, we propose the development of a suspension that exhibits non-Newtonian behaviour in the physiological shear stress regime and gives a good correlation with erythrocyte damage in the high shear stress regime.

\section{Materials and Methods}

\subsection{Microparticle Alginate Gel Material}

Alginate is a polysaccharide derived from sea algae that consists of a linear arrangement of block copolymers of 1-4 linked $\beta$-D-mannuronic (M) acid and $\alpha$-L-guluronic $(\mathrm{G})$ acid. Divalent ions have been found to form crosslinks in alginate by binding the guluronic residues, inducing a stable alginate gel. A commonly used divalent ion is calcium $\left(\mathrm{Ca}^{2+}\right)$. The properties of alginate have been the center of many studies since its introduction in the biomedical field. Its biocompatibility, high water retention, and modifiable mechanical properties have currently many uses in bioengineering. Many of those uses include polymer films, cell encapsulation [15], wound dressing, surgical sponges, endovascular occlusion [16], and color stabilization [17].

Amongst the numerous studies made on the properties of alginate, the ones of interest are its mechanical and diffusive properties. From literature $[18,19]$, the elastic modulus of the alginate gel ranges from 2.31 to $75 \mathrm{kPa}$. This wide range of this alginate property is dependent on its physico-chemical characteristics such as chemical composition, molecular weight, and from which plant family of algae it was extracted. It is also found that these properties will vary according with gelling conditions such as temperature and $\mathrm{pH}$ of alginate solution [20]. Yamagiwa et al led an extensive study of these effects on the properties of interest. This adjustment in mechanical properties is also useful in the development of a blood analog. It was found that the ratio of mannuronic and guluronic acid $(\mathrm{M} / \mathrm{G})$, temperature, and alginate concentration have the greatest effect on the compressive and diffusive properties of alginate gel. However, most of the experiments conducted on these properties were only designed to provide selection guidelines for drug controlled-release applications $[20,21]$ where the size of the drug molecule is very small compared to the pore size of the alginate gel [22]. In Figure 1, we can observe the random pores on an alginate particle. Furthermore, the alginate that is obtained from the distributor (Sigma-Aldrich Inc) is found to have an 
$\mathrm{M} / \mathrm{G}$ ratio of 1.56 which, from [20], would have a minimum diffusion coefficient.

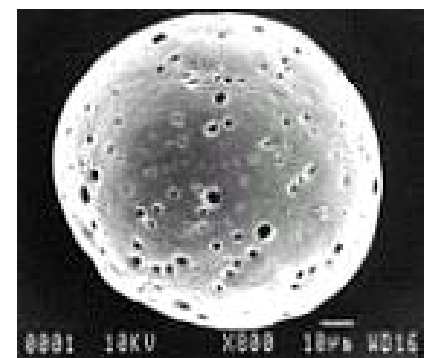

Figure 1. SEM representation of an alginate particle and its pores.

From experiments that use alginate as a color stabilizer for food and clothing [17], the encapsulation of pigments is more suited for our studies since there is little or no diffusion of the color out of the particles. As described in the following, we propose to develop our blood model by incorporating a dye agent inside alginate microparticles.

\subsection{Suspension Solution}

Calcium Chloride, sterile Sodium Alginate (medium viscosity grade, $\mathrm{MW}=38500 \mathrm{kDa}$ ), and sodium citrate were purchased from Sigma-Aldrich (St-Louis, $\mathrm{MO})$. The encapsulated color is a liquid pigment (Phenol Red No.1070) suspended in Polypropylene Glycol obtained from Kama Pigment (Montreal, Canada). The microparticles were prepared using a commercial high-pressure air-atomizing nozzle designed by Turbotak (Toronto, Canada) that was previously employed by Kwokk et al [23]. From a technique originally developed by Lim and Sun [15], the sodium alginate is dissolved in dionized water at $1.2 \%$ (wt.) concentration and stored at room temperature until the polymer completely dissolved. The liquid pigment was added to the aqueous alginate at $1 \%$ (vol.) concentration and stirred until the pigment became homogeneously distributed within the solution. The microparticles were produced by spraying the colored alginate solution in a receptacle containing an aqueous solution of calcium chloride at $1.5 \%$ (wt.) concentration. High pressure and the alginate solution were fed to the nozzle using a commercial air compressor (Jun-air USA Inc., Illinois) and a roller pump (Masterflex L/S, L/S 14 tubing), respectively (see Figure 2). The pressure, pumping speed, and the height of the nozzle were reported to have noticeable influence on the size distribution of the microparticles [23-25]. The most efficient settings to obtain the desired microparticle size were obtained with an applied pressure of 4.5 bar (65 PSI), a flow rate of $2 \mathrm{ml} / \mathrm{min}$, and a nozzle height of $53 \mathrm{~cm}(21 \mathrm{in})$.
Once the entire volume of colored alginate was used, the content of the receptacle was sieved through a 100 microns mesh size followed by a 50 microns mesh size to further reduce the size distribution. The microparticle batch was then re-suspended into smaller $10 \mathrm{ml}$ samples of new $1.5 \%$ (wt.) concentration of calcium chloride solution at a particle volume concentration of $30 \%$.

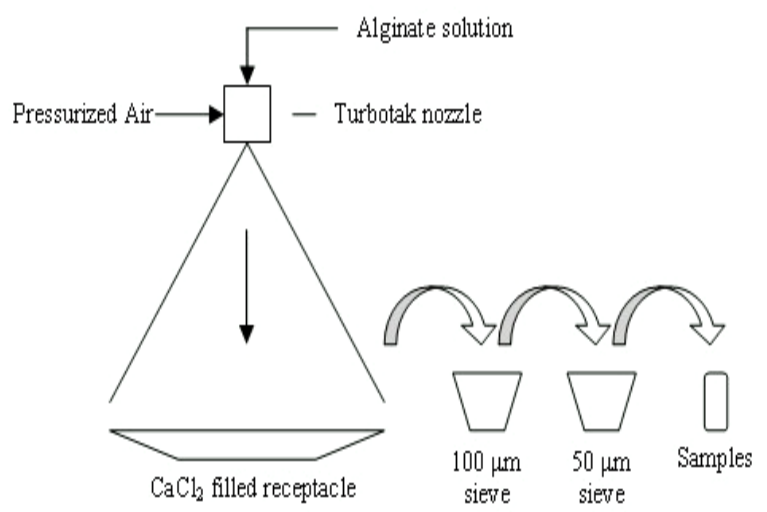

Figure 2a Schematic diagram of the experimental apparatus for producing the microparticles. The pressurized air and the alginate solution are fed into the nozzle and sprayed into the receptacle. The alginate will polymerize upon contact with the aqueous solution containing calcium ions. The suspension is then sieved through two mesh sizes to obtain the desired size distribution.

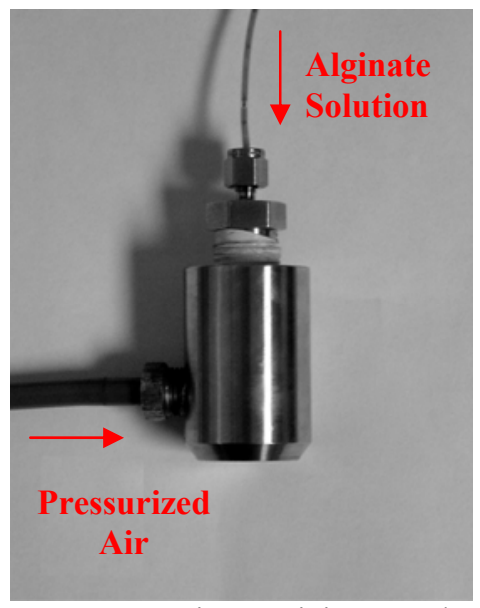

Figure 2b. Air-atomizing nozzle.

\subsection{Physical Properties Measurements}

The dynamic viscosity of the resulting liquid suspensions was measured using a rheometer (Bohlin CVO $120 \mathrm{HR}$ ) with a double gap measurement system. The temperature was controlled by a water bath/circulator (Neslab RTE-111) connected to the rheometer. For the dynamic viscosity experiments, the 
applied shear rates were from $0.01-300 \mathrm{~s}^{-1}$, which is the range normally found in normal blood flows in healthy humans. The samples were pre-sheared for 60 seconds and kept idle for 60 seconds to insure particle homogeneity within the device. Each sample was sheared within the specified range with logarithmic increments every 10 seconds for a total test time of 150 seconds and a total number of 15 data points per test. The tests were conducted at a temperature of 25 ${ }^{\circ} \mathrm{C}$. Each sample preparation was analyzed three times under the same conditions.

\subsection{Shear Device for Hemolysis Experiments}

The shear stresses were applied on the fluid by using a custom-made shear device previously described and tested by Pohl et al [9, 10]. The device consists of an upper rotating plate and a lower static plate. The working principle of the device is displayed in Figure 3(a, b). A fluid volume of $10 \mathrm{ml}$ was forced by a syringe pump system (Sage 180, Sage Instruments) through a circumferential gap measuring $5 \mathrm{~mm}$ in length (distance between the inner and outer annular channels) and $0.140 \mathrm{~mm}$ in height. For the hemolysis experiments, we applied shear rates in the range of 39 - $67428 \mathrm{~s}^{-1}$ (which corresponds to shear stresses of $0.15-269.18 \mathrm{~Pa}$ assuming a constant viscosity of 4 mPa.s at high shear rates) The mathematical relation of the shear rate for the device as a function of angular velocity, channel radius, and channel height is derived from standard parallel plate viscometer relation [30]:

$\dot{\gamma}(R)=\frac{R \Omega}{h}$

where $\dot{\gamma}(R)$ is the shear rate at a particular radial distance $R$ from the center of the disk plates. The variable $h$ represents the gap height and $\Omega$ represents the angular speed at which the upper disk is rotating.

The mean shear rate at which the blood analog will be exposed in the channel is [30]:

$\dot{\gamma}_{\text {mean }}=\frac{1}{\left(R_{o}-R_{i}\right)} \int_{R_{i}}^{R_{o}} \frac{r \Omega}{h} \cdot d r$

where $R_{i}$ and $R_{o}$ are the radial boundaries of the channel.

The integration yields the following relationship:

$\dot{\gamma}_{\text {mean }}=\frac{\left(R_{o}{ }^{2}-R_{i}{ }^{2}\right) \Omega}{2\left(R_{o}-R_{i}\right) h}$

The parameters of our experiments were $R_{i}=27 \mathrm{~mm}$, $R_{o}=32 \mathrm{~mm}, h=0.140 \mathrm{~mm}, \Omega=0.1875-320 \mathrm{rad} / \mathrm{s}$, corresponding to a resulting shear rate range of 39 to $67428 \mathrm{~s}^{-1}$.

The pumping time of the syringe was used to set the exposure time of the fluid in the shear field that ranged from 156-1297 $\mathrm{ms}$ after having calibrated the syringe pump. From the reviewed literature, these exposure times correspond to what can be observed in cardiovascular prostheses [5].

The actual tests were carried at four shear stress increments $(60,120,200$, and $260 \mathrm{~Pa})$ and at four different exposure times $(204,311,648$, and $1297 \mathrm{~ms})$ for a total of 16 tests in duplicate series.
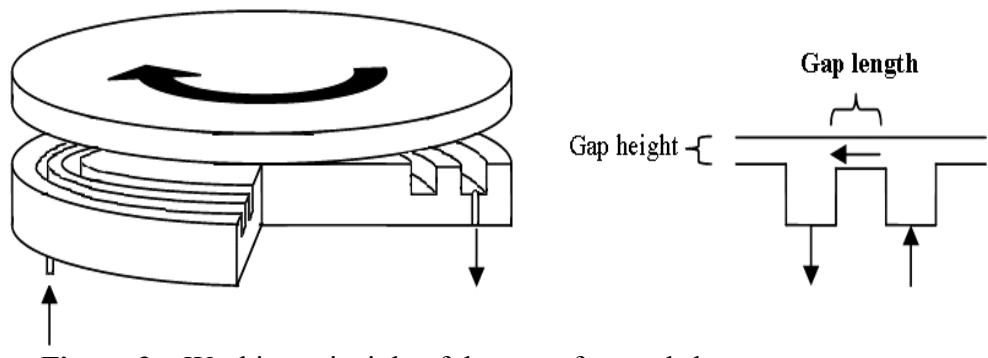

Figure 3a. Working principle of the manufactured shear apparatus.

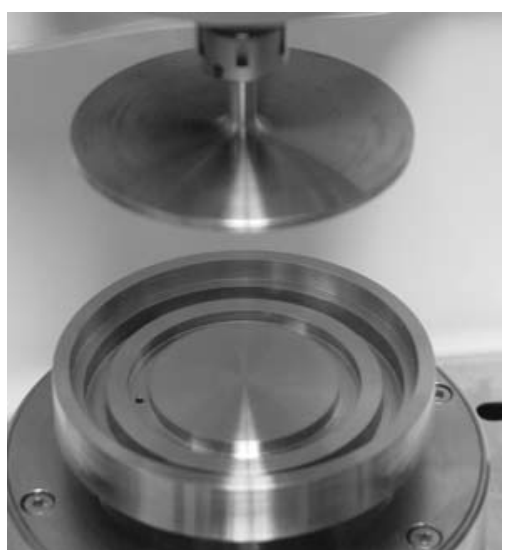

Figure 3b. Actual shear apparatus. 


\subsection{Measures of Blood and Blood Analog Damage}

From the hemolysis study done by Wurzinger et al [4], the result of the damaging effects on erythrocytes is given by the index of hemolysis (HI):

$$
H I=\frac{\Delta H b}{H b} \cdot 100 \%
$$

To quantify the diffusion of the microparticles, half the original concentration of microparticles was dissolved in a solution of sodium citrate, which corresponded to a $50 \%$ hemolysis. The resulting solution was then divided and diluted into 4 samples of different concentrations $(0.1,0.2,0.3$, and $0.4 \%)$. The samples were read with a UV-visible spectrophotometer (Cary 100 Bio, Variant) equipped with its own software package. The readings were done at a common wavelength found on all samples (Figure 4a). A relationship between color intensity and pigment concentration was obtained as displayed in Figure 4b.

$$
D C(\%)=\frac{(A b s+0.10503)}{0.46332}
$$

where $A b s$ is the intensity value measured at the 565 $\mathrm{nm}$ wavelength and $D C$ is the pigment concentration released from the microparticles in percentage.

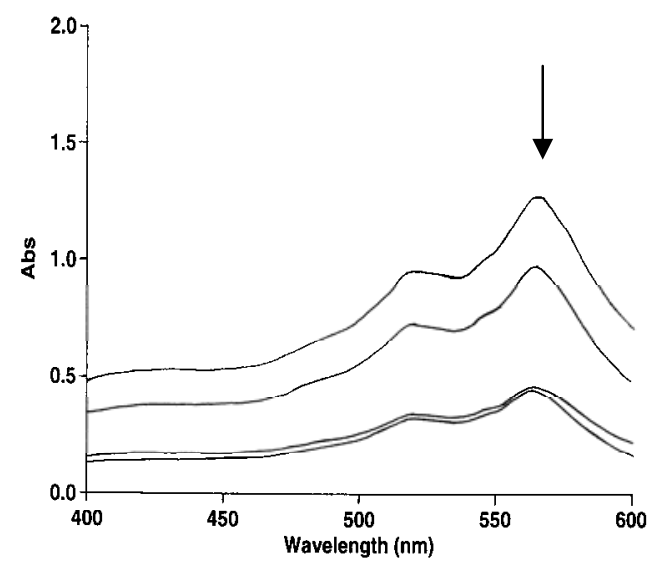

Figure 4a. Typical reading of a sample using the UVspectrophotometer. The readings were made by measuring the absolute value at the $565 \mathrm{~nm}$ wavelength.

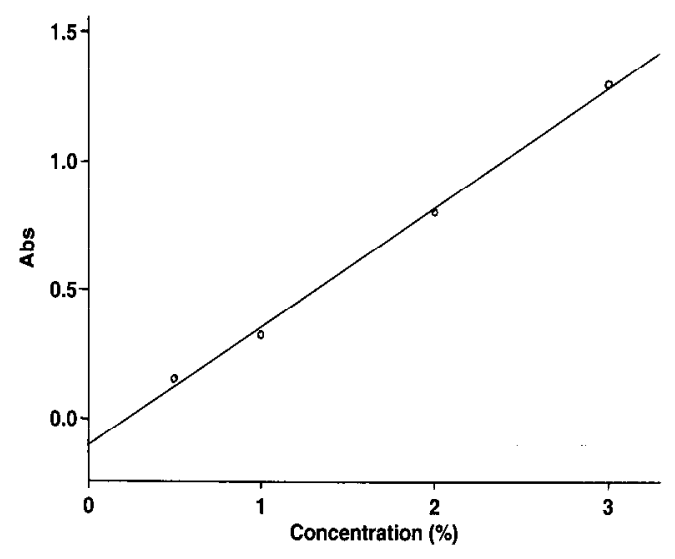

Figure 4b. The resulting relationship between color intensity and pigment concentration at $565 \mathrm{~nm}$.

\section{Results}

\subsection{Particle Size Distribution}

An image processor and a numerical software (TableCurve 2D 5.01, Systat Software Inc.) were used to characterize the microparticle size distribution. A sizing grid was generated with the image processor over the digital image of a sample to enable us to count the particle based on their sizes.

From the distribution, we obtain a microparticle diameter mode of around 17 microns, which is approximately twice that of a red blood cell. The full width half maximum is approximately 19 microns.

\subsection{Dynamic viscosity dependence on shear rate}

The average curve of dynamic viscosity measurements using the methodology described in section 2.3 is displayed in Figure 6 for a $30 \%$ hematocrit suspension in $1.5 \%$ calcium chloride medium. As shown below, this hematocrit was determined using a bisection method in order to match the asymptotic dynamic viscosity of whole human blood at high shear rates. All samples exhibited shear-thinning properties from shear rate of $0.1-150 \mathrm{~s}^{-1}$. At higher shear rates $\left(>150 \mathrm{~s}^{-1}\right)$, a transition to a Newtonian behavior can be seen stabilizing at $\sim 4 \mathrm{mPa}$.s, which is very close to that of blood in this particular range. The average curve corresponding to whole blood was obtained from data found in the literature [31-37].

We note that the general behaviour of the blood analog suspension follows that of average whole human blood. As mentioned above, the asymptotic (Newtonian) viscosity is also very close to that of human blood. 


\subsection{Shear-induced microparticle diffusion}

The particle diffusion results obtained with the apparatus and methodology described in sections 2.4 and 2.5 are summarized in Figure 7. The release of the dye agent from the microparticles is shown to be dependent on the duration of exposure for different applied shear stresses.

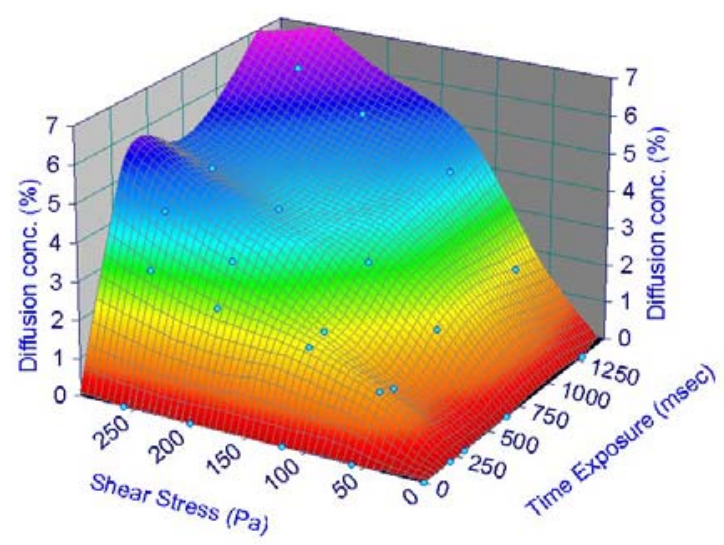

Figure 7. Concentration of dye agent diffused from the microparticles for different duration of exposure and shear stress levels (experimental data shown as circles and fitted with a spline surface)

The relationship between the concentration of dye agent released and the shear-induced hemolysis of human blood found in literature is shown in Figure 8. The following a third power exponential provides an excellent quality fit:

HI $(\%)=0.1194(D C)^{3}, \quad R^{2}=0.98$

The surface regression was done using a mathematical software (Tablecurve 3D v.4.0.01, Systat Software Inc.) to fit a particular mathematical model to the datapoints. For comparison purposes, the same mathematical model used by Wurzinger [4, 6], HI (\%) $=\mathrm{A} \tau^{\alpha} \mathrm{t}^{\beta}$, was used. The parameters for $\mathrm{A}, \alpha$, and $\beta$ for the blood analog and whole human blood are given in Table 1. The surface regression plots with one fixed parameter is to see their influence of the quality of the fit done by the software.

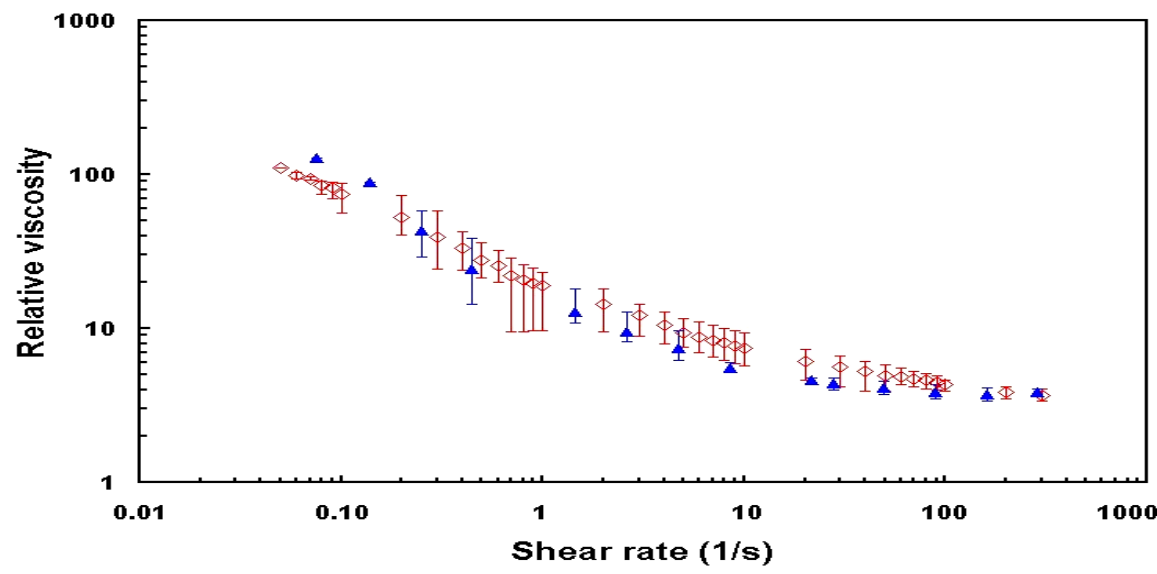

Figure 6. Average dynamic viscosity behaviour of the microparticle suspension $(\mathbf{\Delta})$ within the applied shear rate range compared to that of whole blood $(\diamond)$.

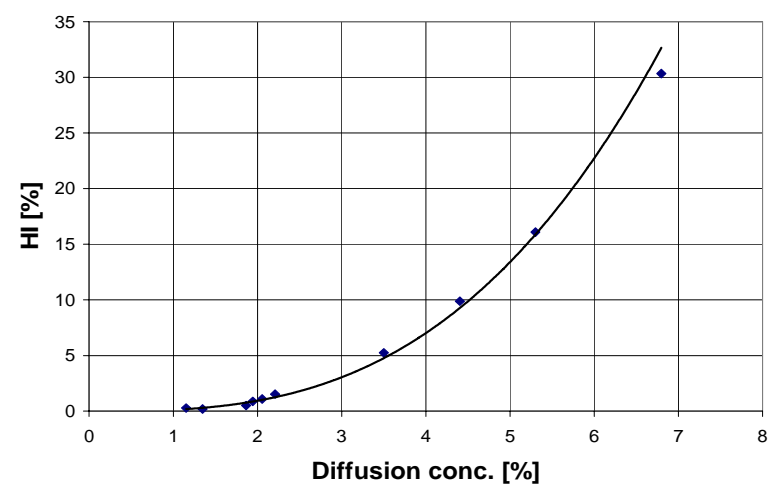

Figure 8. Relationship between the concentration of dye agent of the blood analog released and the shear-induced hemolysis of human blood found in literature. 
Table 1. Parameter comparison for the surface fit for the experimental datapoints.

\begin{tabular}{|c|c|c|c|c|}
\hline Parameters & $\mathbf{A}$ & $\alpha$ & $\beta$ & Comments \\
\hline Wurzinger et al & $3.65 \times 10^{-5}$ & 0.785 & 2.416 & $\begin{array}{c}{[4,6]} \\
\text { (Figure 9) }\end{array}$ \\
\hline Present Study & $2.03 \times 10^{-3}$ & 1.222 & 1.659 & $\mathrm{R}^{2}=0.99$ \\
\hline $\begin{array}{c}\text { Present Study } \\
\text { ( } \beta \text { fixed at 2.416) }\end{array}$ & $3.38 \times 10^{-5}$ & 0.953 & 2.416 & $\mathrm{R}^{2}=0.98$ \\
\hline $\begin{array}{l}\text { Present Study } \\
\text { ( } \text { a fixed at } 0.785 \text { ) }\end{array}$ & - & 0.785 & - & No fit convergence \\
\hline $\begin{array}{c}(\beta \text { fixed at } \\
2.416) \\
(\alpha \text { fixed at } \\
0.785)\end{array}$ & $3.40 \times 10^{-5}$ & 0.785 & 2.416 & $\mathrm{R}^{2}=0.95$ \\
\hline
\end{tabular}

Table 2. Global error from the parameter vectors of the surface fits of the experimental data points compared to the parameters obtained by Wurzinger et al $[4,6]$.

\begin{tabular}{|c|c|c|c|c|}
\hline Parameters & $\mathbf{A}$ & $\alpha$ & $\beta$ & $\begin{array}{c}\text { Global Error } \\
(\%)\end{array}$ \\
\hline $\begin{array}{c}\text { Present } \\
\text { Study }\end{array}$ & $\underset{3}{2.03 \times 10^{-}}$ & 1.222 & 1.659 & 30.4 \\
\hline $\begin{array}{c}\text { Present } \\
\text { Study } \\
(\beta \text { fixed at } \\
2.416)\end{array}$ & $3.38 \times 10^{-}$ & 0.953 & 2.416 & 6.61 \\
\hline $\begin{array}{c}(\beta \text { fixed at } \\
2.416) \\
(\alpha \text { fixed at } \\
0.785)\end{array}$ & $3.40 \times 10^{-}$ & 0.785 & 2.416 & $\sim 0$ \\
\hline
\end{tabular}

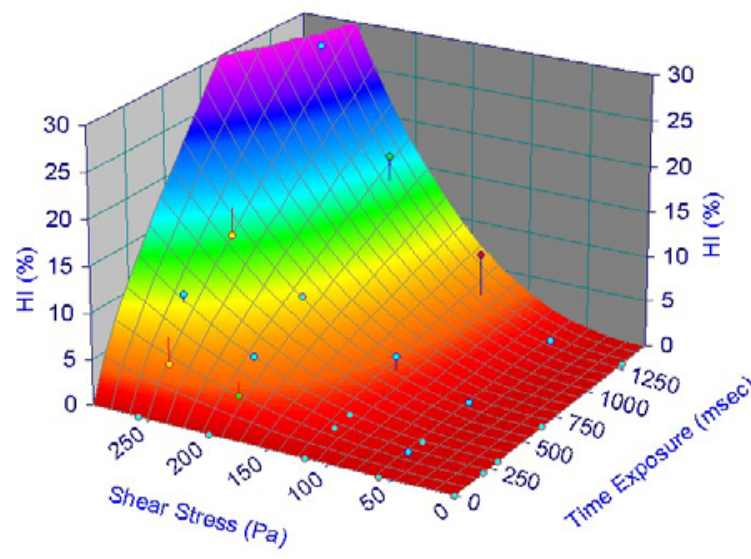

Figure 9. Correlated data points comparison with the surface plot from literature $[4,6]$.

The global error of the surface regression plots is calculated by the Euclidian Method on the parameter vector $[A, \alpha, \beta]$ which is expressed by the following relationship:

$$
\operatorname{Error}(\%)=\sqrt{\frac{\left(A_{2}-A_{1}\right)^{2}+\left(\alpha_{2}-\alpha_{1}\right)^{2}+\left(\beta_{2}-\beta_{1}\right)^{2}}{\left(A_{1}^{2}+\alpha_{1}^{2}+\beta_{1}^{2}\right)}} \times 100 \%
$$

where $A, \alpha, \beta$ are the parameters from the mathematical model used by Wurzinger et al and to fit the experimental data points. The resulting error for the surface plots are given in Table 2.

\section{Discussion}

We have presented a procedure to manufacture a blood analog suspension of microparticles that can reproduce the non-Newtonian properties of human whole blood at physiological shear rates such as shear- 
thinning. Furthermore, the suspension can also be used for hemolysis experiments. Such properties in an analog would be useful in a wide range of applications to study blood flow and hemolytic properties associated with the use of a medical device. It would reduce the need to employ whole blood from various species such as bovine, pigs, etc., reduce the risks of infection and contamination to the users, and standardize the conditions of the tests and provide for a stable, synthesized fluid.

\subsection{Dynamic viscosity dependence on shear rate}

The non-Newtonian effect at applied shear rates is due to the effect of two major properties of the suspension. One is attributed to the fact that a suspension is generally non-Newtonian shear-thinning and the second is the presence of $\mathrm{Ca}^{2+}$ ions that contribute to the aggregation of the micoparticles. From literature [26], once the crosslink between the $\mathrm{Ca}^{2+}$ and alginic acid is made, there are still unlinked carboxylic binding sites where loose calcium ions can bind and unbind randomly. It is assumed that the presence of additional $\mathrm{Ca}^{2+}$ ions produces a bridging effect between two or more particles. The effects of a change in calcium concentration or a change of ions having a higher affinity such as barium $\left(\mathrm{Ba}^{2+}\right)$ and Strontium $\left(\mathrm{Sr}^{2+}\right)$ have not been covered in this work.

Although it was not thoroughly studied in the present work, the presence of $\mathrm{Na}^{+}$ions may also contribute to the reduction of the elastic modulus of the microparticles [26] of about $63 \%$. A reduction the elastic modulus may contribute in a reduction of viscosity at shear rates $>100 \mathrm{~s}^{-1}$ (increased elastic deformation of the particles).

Another factor that should be accounted for the viscosity is the particle size distribution. Current accessible technology to produce microparticles does not permit a near homogeneous distribution for sizes below 160 microns [22]. A suspension having an homogeneous size distribution of microparticles might produce more uniform properties and behaviours. Furthermore, relationships with current mathematical models can be established more easily in order to predict the behaviour of the suspensions in flow.

\subsection{Shear-induced microparticle diffusion}

The diffusive properties of the alginate microparticles were used to reproduce the hemolytic potential of red blood cells. To the best of our knowledge, no experiments have attempted to model the diffusive properties of alginate loaded with a dye agent under the coordinated influence of shear stress and duration of exposure, this work provides a good foundation for future studies and match them with whole human blood properties. Compared to the works done by Pohl et al $[9,10]$, the diffusion of a dye agent from microparticles provide a more natural and similar behaviour of RBC damage than the molecular degradation of APA. Furthermore, microparticles can also be used to understand how the RBCs are transported and exposed to shear stress.

From Table 2, it can be observed that there is noticeable difference between the parameters from Wurzinger and the blood analog with a global parameter error of $30 \%$. However, the surface regression fit with one fixed parameter obtained a global error of less than $6 \%$, which gives a very close approximation of the parameters derived by Wurzinger et al. Thus, fixing a parameter at 2.416 can be used to give a satisfactory relation between the concentration of released dye from the blood model to the concentration of released hemoglobin from human blood. From Figure 7 we have noticed a premature release of dye agent in the shear stress range of 0-120 $\mathrm{Pa}$ similar to a burst release often observed in literature [27]. This initial release cause higher values to be recorded at low shear stresses as opposed to smaller values seen in Figure 9 Adding a layer of Chitosan, a biological polymer, to the alginate microparticle can reduce the pore size to a range of 20 to $100 \mathrm{kDa}$ in contrast to $\sim 1000 \mathrm{kDa}$ for alginate alone. This reduction of pore size may decrease the release of dye agent in the low shear stress range.

As mentioned earlier, the current apparatus used to create the microparticles does not enable us to create perfectly spherical and homogeneously-sized beads. If this design problem is resolved, the alginate microparticles could serve as the initial step in the development of Alginate-Poly-L-Lysine-Alginate microcapsules, a commonly-used microcapsule in the biomedical field. This concept of a liquid core encapsulated by an elastic polymeric membrane has been the subject of several papers [26]. However, a critical factor of the protocols is related to the generation of spherical alginate microparticle since the morphology has a significant influence on the integrity of the membrane. For example, a dropletshaped microparticle would develop a weaker membrane thickness at the tail that would break once the alginate core is liquefied. The microcapsule would be a more natural model to reproduce the hemolytic potential of red blood cells, as compared to the diffusive properties of standard alginate microparticles. The polymeric membrane mechanical properties can also be characterized and be mathematically modeled by known mathematical models developed by other groups such as BarthèsBiesel and Drochon [28, 29]. These models can help 
us find the correct membrane properties to reflect the RBC's.

The use of other polymers such as PLGA may also yield different diffusive properties as alginate and might prove to be better blood analogs. However, the choice of alginate was made in terms of production simplicity whereas other polymers often need more complicated and costly preparation protocols and different chemicals to produce the same microparticles.

Another factor to take into account is the size distribution of the microparticles. Larger particles are more likely to deform under stress where smaller ones are more rigid. This may force more dye agent from the larger particle yielding a higher dye concentration compared to more homogenously-sized beads.

We have demonstrated that a model blood consisting of natural gel microparticles can reproduce the same shear-thinning properties as human blood in the physiological range of shear rates. Another property that has been satisfactorily matched is the hemolytic potential of human blood at high shear stresses. Although the blood analog did not exactly reproduce the hemolytic properties of blood, its general properties are sufficiently similar to be employed as a preliminary evaluation tool for bloodwetted devices known to cause red blood cell damage.

\section{References}

[1] Rand RP (1964), Mechanical Properties of Red Cell Membrane. Part 2: Viscoelastic Breakdown of the Membrane, Biophys. J., 4, pp. 303-316

[2] Papantonis D (1991), Numerical Prediction of the Shear Stresses and the Mean Exposure Time for Radial Flow Impellers, In: Schima H, Thoma H, Wieselthaler G, Wolner E, eds. Proceedings of the International Workshop on Rotary Blood Pumps, Vienna, pp. 63-69

[3] Lambert J. (1976), Die Hämolysierende Wirkung Hoher, Kurzzeitiger Schubspannungen, $\mathrm{PhD}$ thesis, Fakultät für Maschinenwesen der EWTH Aachen, Aachen, Germany.

[4] Wurzinger LJ, Optiz R, Eckstein H (1986), Mechanical BloodTrauma: An Overview, Angéiologie, 38(3), pp. 8197

[5] Heuser G, Opitz R (1980), A Couette Viscometer for Short Time Shearing of Blood, Biorheology, 17, pp. 1724

[6] Giersiepen M, Wurzinger LJ, Optiz R, Reul H (1990), Estimation of Shear Stress-related Blood Damage in Heart Valve Prostheses - In Vitro Comparison of 25 Aortic alves, Int. J. Artif. Org., 13(5), pp. 300-306
[7] Paul R, Apel J, Klaus S, Schügner F, Schwindke P, Reul H (2003), Shear Stress Related Blood Damage in Laminar Couette Flow, Artif. Org., 27(6), pp. 517-529

[8] Maruyama O, Yamane T, Tsunemoto N, Nishida M, Tsutsui T, Jikuya T (1999), A preliminary Study of Microcapsule Suspension for Hemolysis Evaluation of Artificial Organs, Artif. Organs, 23(3), pp, 274-279

[9] Pohl M, Samba O, Wendt MO, Vlastos GA (1998), Shear Stress Related Hemolysis and its Modelling by Mechanical Degradation of Polymer Solutions, Int. J. Artif. Org., 21(2), pp. 107-113

[10] Pohl M, Wendt MO, Koch B, Vlastos GA (2000),Mechanical Degradation of Polyacrylamide Solutions as a Model for Flow Induced Blood Damage in Artificial Organs, Biorheology, 37, pp. 313-324

[11] Jikuya T, Tsutsui T, Shigeta O, Sankai Y, Mitsui T (1998) Species Difference in Erythrocyte mechanical Fragility : Comparison of Human, Bovine, and Ovine Cells, ASAIO J., M452-455

[12] Mueller MR, Schima H, Engelhardt H, Salat A, Olson DB, Losert U, Wolner E (1993), In vitro hematological testing of Rotary Blood Pumps: Remarks in Standardization and Data Interpretation, Artif. Organs, 17(1), pp. 103-110

[13] Thurston GB (1996), Viscoelastic Properties of Blood and Blood Analogs, Advances in Hemodynamics and Hemorheology, 1, pp. 1-30

[14] Maruyama O, Yamane T, Mishida M, Aouidef A, Tsutsui T, Jikuya T, Masuzawa T (2002), Fractural Characteristic Evaluation of a Microcapsule Suspension Using a Rotational Shear Stressor, ASAIO J., pp. 365-373

[15] Lim F, Sun AM (1980), Microencapsulated islet as bioartificial endocrine pancreas, Science, 210, pp. 908-910

[16] Becker TA, Kipke DR (2002), Flow Properties of Liquid Calcium Alginate Polymer Injected Through Medical Microcatheters for Endovascular Embolization, J. Biomed. Mater. Res., 61(4), pp. 533-540

[17] Saito K, Murata T, Mori T (1995), Encapsulation of Carthamin and Safflor Yellow B in Calcium Alginate Beads - Techniqcal Stabilization of the Colours, Int. J. Food Sci Tech., 29, pp. 715-719

[18] Leroux MA, Guilak F, Setton LA (1999), Compressive and Shear Properties of Alginate Gel: Effects of Sodium Ions and Alginate Concentration, J. Biomed. Mater. Res., 47(1), pp. 46-53

[19] Ohba K, Ando T, Yoza I, Onoue A, Uragami U, Miyata T (2002), Model Blood Consisting of Dense Suspension of Natural Polymeric Gel Particles, World Biomechanics Congress 2002 Poster Presentation, Calgary, Canada, No. 70 
[20] Yamagiwa K, Kowazw T, Ohkawa A (1995), Effect of Alginate Composition and Gelling Conditions on Diffusional and Mechanical Properties of Calcium-Alginate beads, J. Chem. Eng. Jap., 28(4), pp. 462-467

[21] Shu XZ, Zhu KJ (2002), The Release Behavior of Brilliant Blue From Calcium-Alginate Gel Beads Coated by Chitosan: The Preparation Method Effect, Eur. J. Pharm. Biopharm., 53, pp. 193-201

[22] notech Website-Encapsulator

(2003),

http://www.inotech.ch/faqs.htm

[23] Kwok KK, Groves MJ, Burgess DJ (1991), Production of 5-15 $\mu \mathrm{m}$ Diameter Microcapsules by an Air-Atomization Technique, Pharm. Res., 8(3), pp. 341-344

[24] Abraham SM, Vieth RF, Burgess DJ (1996), Novel Technology for the Preparation of Sterile Alginate-Poly-LLysine Microcapsules in a Bioreactor, Pharm, Dev. Technol., 1, pp. 63-68

[25] Cui JH, Goh JS, Park SY, Kim PH, Lee BJ (2001), Preparation and Physical Characterization of Alginate Microparticles Using Air Atomization Method, Drug Dev. Indus. Pharm, 27(4), pp. 309-319

[26] Gugerli R, Cantana E, Heinzen C, Von Stockar U, Marison IW (2002), Quantitative Study of the Production and Properties of Alginate/Poly-L-Lysine Microcapsules, J. Microencapsulation, 19(5), pp. 571-590

[27] Huang, X. \& Brazel, C.S. On the importance and mechanism of burst release in matrix-controlled drug delivery systems J Control Release 73, 121-136 (2001)

[28] Barthes-Biesel D. (1993), Theoretical modelling of the motion and deformation of capsules in shear flows, Biomater Artif Cells Immobilization Biotechnol., 21(3), pp. 359-373.
[29] Drochon A, Barthes-Biesel D, Lacombe C, Lelievre JC. (1990), Determination of the red blood cell apparent membrane elastic modulus from viscometric measurements., J Biomech Eng., 112(3), pp. 241-9. Erratum in: J Biomech Eng (1991), 113(1), p. 103.

[30] Whorlow RW (1992), Rheological Techniques, Ellis Horwood Series in Physics and its Applications, Ellis Horwood Limited, England, pp. 118-119

[31] Brooks DE, Goodwin JW, Seaman GVF (1970), Interactions Among Erythrocytes Under Shear, J. Applied Physiol., 28(2), pp. 172-177

[32] Cokelet GR (1972), The Rheology of Human Blood In: Fung YC, Perrone N, Anliker M, eds. Biomechanics. Englewood Cliffs, NJ, Prentice-Hall, p. 63

[33] Chien S, Usami S, Dellenback RJ, Bryant CA (1971), Comparative Hemorheology-Hematological Implications of Species Difference in Blood Viscosity, Biorheology, 8(35), pp. 35-57

[34] Chien S, Usami S, Taylor M, Lundberg JL, Gregersen MI (1966), Effects of Hematocrit and Plasma Proteins of Human Blood Rheology at Low shear Rates, J. Appl. Physiol., 21, pp. 81-87

[35] Chien S, Usami S, Dellenback RJ, Gregersen MI (1970), Shear Dependent Deformation of Erythrocytesd in Rheology of Human Blood, Am. J. Physiol., 219, pp. 136142

[36] Merrill EW, Gilliland ER, Cokelet GR, Shin H, Britten A, Wells RE (1963), Rheology of Human Blood, Near and at Zero Flow, Biophys. J, 3, pp. 199-213

[37] Shin S, Keum DY (2002), Measurement of Blood Viscosity Using Mass-Detecting Sensor, Biosensors \& Bioelectronics, 17, pp. 383-388 